\title{
A Closer Look
}

\section{Pythagoras: Everyone knows his famous theorem, but not who discovered it 1000 years before him}

\begin{abstract}
Bruce Ratner
is a PhD and founder and President of DM STAT-1 Consulting. DM STAT-1 specializes in the full range of standard statistical techniques, and methods using hybrid machine learning-statistics algorithms, such as its patented GenIQ Model (C Modeling and Data Mining Software, accross a wide range of industry sectors and business functions. Bruce is the author of the best-selling book Statistical Modeling and Analysis for Database Marketing: Effective Techniques for Mining Big Data (based on Amazon Sales Rank since June 2003). He is an often-invited speaker at public and private industry events.
\end{abstract}

\begin{abstract}
Everyone who has studied geometry can recall, well after the high school years, some aspect of the Pythagorean Theorem. However, the story of Pythagoras and his famous theorem is not well known. Some of the plot points of the story are presented in this article. The famous theorem goes by several names, some grounded in the behavior of the day, including the Pythagorean Theorem, Pythagoras' Theorem and notably Euclid I 47. The Pythagorean Theorem is arguably the most famous statement in mathematics, and the fourth most beautiful equation. There are well over $\mathbf{3 7 1}$ Pythagorean Theorem proofs, originally collected and put into a book in 1927, which includes those by a 12-year-old Einstein (who uses the theorem two decades later for something about relatively), Leonardo da Vinci and President of the United States James A. Garfield. Pythagoras is immortally linked to the discovery and proof of a theorem that bears his name - even though there is no evidence of his discovering and/or proving the theorem. There is concrete evidence that the Pythagorean Theorem was discovered and proven by Babylonian mathematicians 1000 years before Pythagoras was born.
\end{abstract}

Journal of Targeting, Measurement and Analysis for Marketing (2009) 17, 229-242. doi:10.1057/jt.2009.16

The purpose of this article is to plot a fascinating story in the history of mathematics. The 4000-year-old story of Pythagoras and his famous theorem is worthy of recounting - even for the math-phobic readership. It is more than a math story, as it tells a history of two great civilizations of antiquity rising to prominence 4000 years ago, along with historic and legendary persons, who not only define the period, but whose individual life stories are quite engaging.

Correspondence: Bruce Ratner

DM STAT-1 Consulting, 574 Flanders Drive,

North Woodmere, NY 11581, USA

E-mail: br@dmstat1.com
Moreover, the theorem seemingly has no ending, as every year students, academicians and problem solvers with a mathematical bent tackle the theorem in an attempt to add new and innovative proofs. In addition, a 350-year-old generalized version of the Pythagorean Theorem, which was proposed by an amateur mathematician, was finally solved, and made the front-page of the New York Times in 1993.

\section{BRIEF BIOGRAPHY OF PYTHAGORAS}

The Greek mathematician Pythagoras has high name recognition, not only in the history of mathematics. Everyone who has studied geometry 
can recall, well after the high school years, some aspect of the Pythagorean Theorem. However, the story of Pythagoras and his famous theorem is not well known. Some story plot points are: the famous theorem goes by several names grounded in the behavior of the day (discussed later in the text), including the Pythagorean Theorem, Pythagoras' Theorem and notably Euclid I 47. The Pythagorean Theorem is arguably the most famous statement in mathematics, and the fourth most beautiful equation. ${ }^{1,2}$ There are well over 371 Pythagorean Theorem proofs originally collected by an eccentric mathematics teacher, who put them in a 1927 book, which includes those by a 12-year-old Einstein, Leonardo da Vinci (a master of all disciplines) and President of the United States James A. Garfield. ${ }^{3-5}$

Pythagoras' likeness in pictures and sculptures, as shown in Figure 1, appears in all geometry textbooks, and books about the history of mathematics. However, ironically, not much is really known about him - not even his likeness. What is known about Pythagoras is generally considered more fiction than fact, as historians who lived hundreds of years later provided the facts about his life. There are definite details of Pythagoras' life from early biographies that use original sources, yet are written by authors who

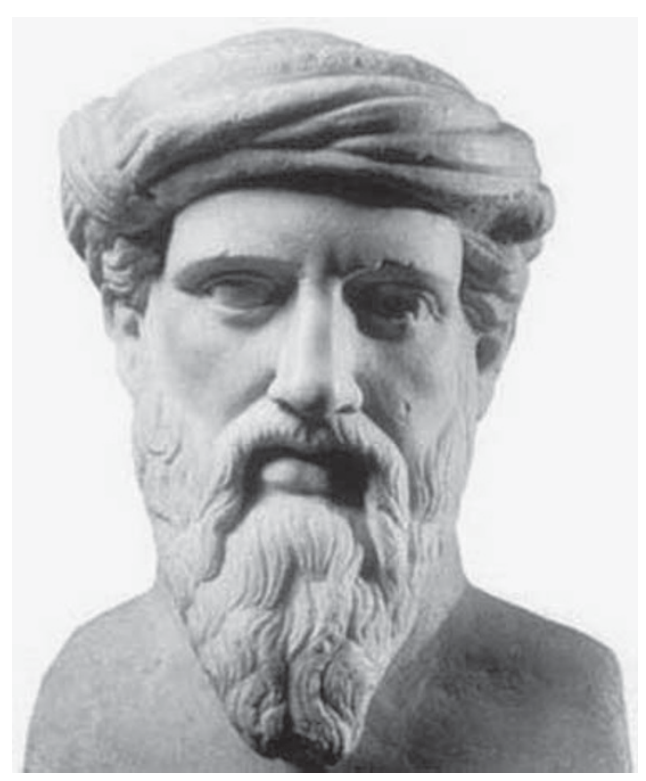

Figure 1: Pythagoras. attribute divine powers to him, and present him as a deity figure. Consequently, most historians treat this information as legend. Few historians view the information with any degree of historical importance because it is obtained from rare original sources. ${ }^{6}$

Historians generally agree that Pythagoras of Samos (born circa 569 BC in Samos, Ionia and died circa $475 \mathrm{BC}$ ) was the first mathematician. $\mathrm{He}$ is an extremely important figure in the development of mathematics, yet relatively little is known about his mathematical achievements. Unlike many later Greek mathematicians, who wrote a number of books, there are no writings by Pythagoras. However, there is evidence that Pythagoras founded a school (in what is now Crotone, to the east of the heel of southern Italy) named the Semicircle of Pythagoras - half-religious and half-scientific, which followed a code of secrecy. Because secrecy is often controversial, Pythagoras is a mysterious figure. The members of the Semicircle of Pythagoras - the Pythagoreans - were bound by an allegiance that was strictly enforced. However, the Semicircle was more than just a school that studied intellectual disciplines, including in particular philosophy, mathematics and astronomy. ${ }^{7}$ The scientific dimension of the school treated numbers in ways similar to the Jewish mysticism of Kaballah, where each number has divine meaning and combined numbers reveal the mystical worth of life. The latter is reflected in the Pythagorean motto: Number Rules the Universe. ${ }^{6}$ The religious dimension of the school included diverse lectures held by Pythagoras attended by men and women, even though the law in those days forbade women from being in the company of men. Pythagoreans consumed vegetarian dried and condensed food and unleavened bread (as matzos, used by the Biblical Jewish priestly class (the Kohanim), and used today during the Jewish holiday of Passover). ${ }^{8}$

One reason for the rarity of Pythagoras original sources was that Pythagorean knowledge was passed on from one generation to the next by word of mouth, as writing material was scarce. Moreover, out of respect for their leader, many of the discoveries made by the Pythagoreans were attributed to Pythagoras himself; this would 
account for the term 'Pythagoras' Theorem'. Consequently, of Pythagoras' actual work nothing is known. On the other hand, his school practiced collectivism, making it hard to distinguish between the work of Pythagoras and that of his followers; this would account for the term 'Pythagorean Theorem'. Therefore, the true discovery of a particular Pythagorean result may never be known. Regardless of the uncertainty of Pythagoras' actual contributions, however, his school made outstanding contributions to mathematics.

Is seems that Pythagoras was the first person to define the consonant acoustic relationships between strings of proportional lengths. Specifically, strings of equal tension of proportional lengths create tones of proportional frequencies when plucked. For example, a string that is 2 feet long will vibrate $x$ times per second (that is, hertz, a unit of frequency equal to one cycle per second), while a string that is 1 foot long will vibrate twice as fast: $2 x$. Furthermore, those two frequencies create a perfect octave. ${ }^{9}$

The most important discovery of Pythagoras' school was the fact that the diagonal of a square is not a rational multiple of its side. ${ }^{10}$ This result proved the existence of irrational numbers. ${ }^{11}$ This finding greatly disturbed the Pythagoreans, as it was inconsistent with their divine belief in numbers: whole numbers and their ratios, which account for geometrical properties, were challenged by their own result. The Pythagoreans were so troubled over the finding of irrational numbers that they swore each other to secrecy about its existence. It is known that one Pythagorean did tell someone outside the school, and he was never to be found thereafter, that is, he was murdered, as Pythagoras himself was murdered by oppressors of the Semicircle of Pythagoras. ${ }^{12}$

\section{THE BABYLONIANS}

Babylonia was situated in an area known as Mesopotamia (Greek for 'between the rivers'). Mesopotamia (arrow 1 in Figure 2) was in the Near East in roughly the same geographical position as modern Iraq. ${ }^{13}$ Two great rivers flowed through this land: the Tigris and the Euphrates (arrows 2 and 3, respectively, in
Figure 2). Mesopotamia was one of the great civilizations of antiquity, rising to prominence 4000 years ago. Thousands of clay tablets, found over the past two centuries, confirm a people who kept accurate records of astronomical events, and who excelled in the arts and literature. Only a small fraction of this vast archeological treasure trove has been studied by scholars. The great majority of tablets lie in the basements of museums around the world, awaiting their turn to be deciphered and to provide a glimpse into the daily life of ancient Babylon. ${ }^{14}$

Among the tablets that have received special scrutiny is that with the identification 'YBC 7289', shown in Figure 3, which represents the tablet numbered 7289 in the Babylonian Collection of Yale University. ${ }^{15}$ The tablet dates from the Old Babylonian period, roughly 1800-1600 BCE, and shows a tilted square and its two diagonals, with some marks engraved along one side and under the horizontal diagonal. The marks are in wedge-shaped characters, carved with a stylus into a piece of soft clay that was then dried in the sun or baked in an oven. They turn out to be numbers, written in the Babylonian numeration system that used the base 60. In this sexagestimal system, numbers up to 59 were written in essentially the modern base-10 numeration system, but without a zero. Units were written as vertical Y-shaped notches, while tens were marked with similar notches written horizontally. ${ }^{15}$

A fortuitous event: the find of tablet YBC 7289 was translated by Dennis Ramsey and dating to YBC 7289 , circa 1900 BC:

4 is the length and 5 is the diagonal. What is the breadth? Its size is not known. 4 times 4 is 16 . And 5 times 5 is 25 . You take 16 from 25 and there remains 9. What times what shall I take in order to get 9 ? 3 times 3 is 9.3 is the breadth. ${ }^{15}$

The number along the upper left side is easily recognized as 30 . The number immediately under the horizontal diagonal is $1 ; 24,51,10$ (this is the modern notation for writing Babylonian numbers, in which the commas separate the sexagesition 'digits', and a semicolon separates the integral part of a number from its 


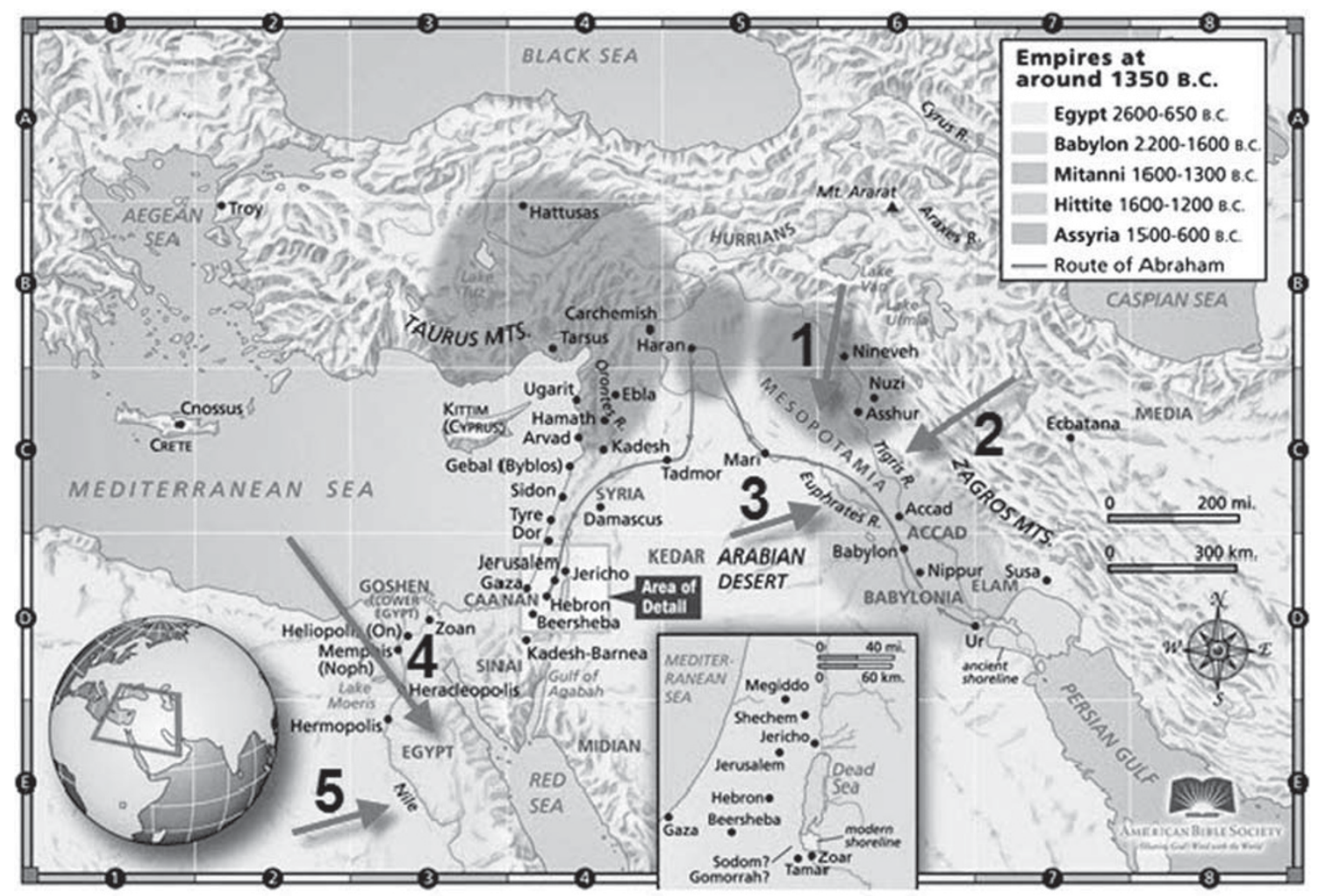

Figure 2: Ancient Near East Empires around 1350 BC

(Egypt, Babylon, Mitanni, Hittite, Assyria, plus Route of Abraham).

fractional part). Writing this number in the base- 10 system, one gets $1+24 / 60+51 / 60^{2}+10 /$ $60^{3}=1.414213$, which is nothing other than the decimal value of the square root of 2 , accurate to the nearest one hundred thousandth.

The conclusion is inescapable. The Babylonians knew the relation between the length of the diagonal of a square and its side: $d=$ square root of 2. This was probably the first number known to be irrational. However, this in turn means that they were familiar with the Pythagorean Theorem - or, at the very least, with its special case for the diagonal of a square $\left(d^{2}=a^{2}+a^{2}=2 a^{2}\right)$ - more than a thousand years before the great sage for whom it was named. The square root of 2, known as Pythagoras' constant, is the positive real number that, when multiplied by itself, gives the number 2 (see Figures 3 and 4)..$^{16,17}$

Two factors with regard to this tablet are particularly significant. First, it proves that the Babylonians knew how to compute the square

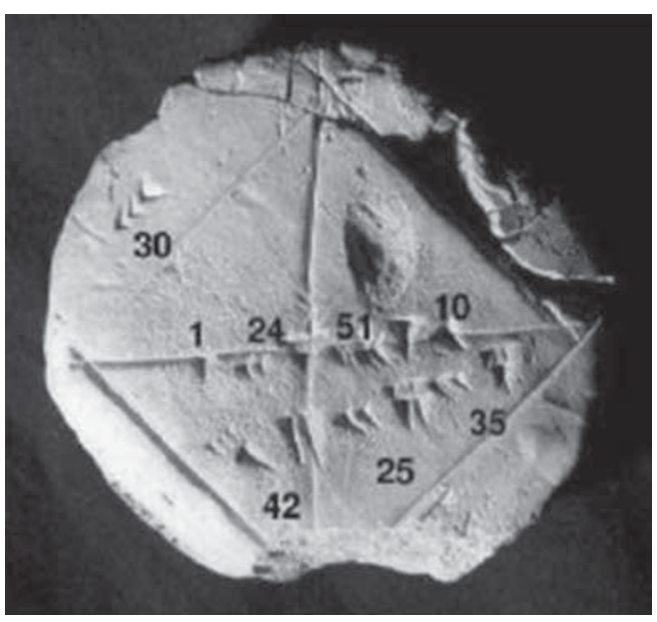

Figure 3: YBC 7289.

root of a number with remarkable accuracy. The unknown scribe who carved these numbers into a clay tablet nearly 4000 years ago showed a simple method of computing: multiply the side of the square by the square root of 2 . But there remains 


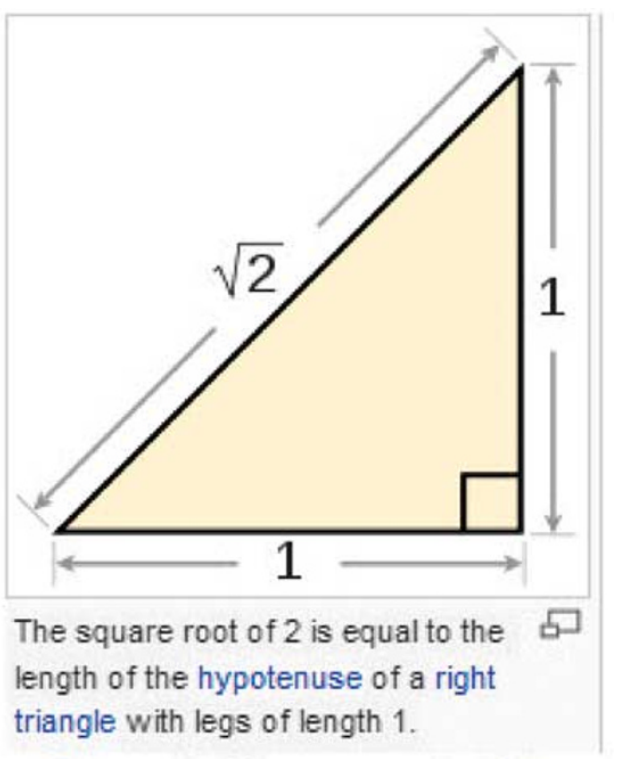

Figure 4: Square root of 2.

one unanswered question: Why did the scribe choose a side of 30 for his example? Probably, 30 was used for convenience, as it was part of the Babylonian system of sexagesimal, a base-60 numeral system. From this one derives the modern day usage of 60 seconds in a minute, $60 \mathrm{~min}$ in an hour and $360(60 \times 6)$ degrees in a circle. ${ }^{18}$

Today, the Pythagorean Theorem is thought of as an algebraic equation, $a^{2}+b^{2}=c^{2}$; but this is not how Pythagoras viewed it. To Pythagoras it was a geometric statement about areas. It was with the rise of modern algebra, circa $1600 \mathrm{CE}$, that the theorem assumed its familiar algebraic form.

Formally, the Pythagorean Theorem is stated in terms of area:

In any right triangle, the area of the square whose side is the hypotenuse (the side opposite the right angle) is equal to the sum of the areas of the squares whose sides are the two legs (the two sides that meet at a right angle). An area interpretation of this statement is shown in Figure $5 .{ }^{19}$

The theorem is usually summarized as follows:

The square of the hypotenuse of a right triangle is equal to the sum of the squares on the other two sides.

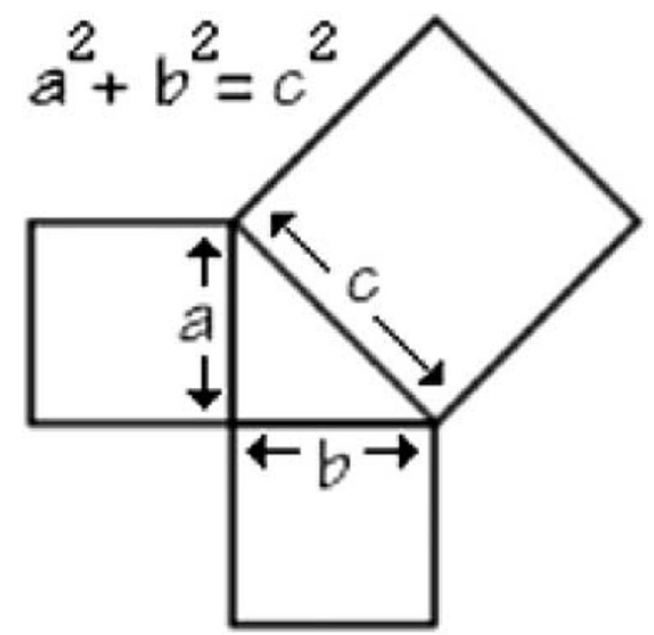

Figure 5: Area version of Pythagoras' Theorem.

\section{A PEOPLE WHO USED THE PYTHAGOREAN THEOREM?}

Ancient Egyptians (arrow 4, in Figure 2), concentrated along the middle to lower reaches of the Nile River (arrow 5, in Figure 2), were a people in Northeastern Africa. The ancient civilization of the Egyptians thrived 500 miles to the southwest of Mesopotamia. The two nations coexisted in relative peace for over 3000 years, from circa 3500 BCE to the time of the Greeks. As to the claim that the Egyptians knew and used the Pythagorean Theorem in building the great pyramids, there is no evidence to support this claim.

Egypt has over 100 pyramids, most built as tombs for their country's Pharaohs. I would be remiss if I did not include an image of the iconic Egyptian Pharaoh Tutankhamen, aka King Tut (Figure 6). Egypt (arrow 4, in Figure 2) and its pyramids are as immortally linked to King Tut as are Pythagoras and his famous theorem. King Tut ruled from the age of 8 for 9 years, 1333-1324 BC. $\mathrm{He}$ was born in $1341 \mathrm{BC}$ and died (some believe he was murdered) in $1323 \mathrm{BC}$ at the age of $18 .^{20}$

\section{THE TEACHER WHO COLLECTED PYTHAGOREAN THEOREM PROOFS}

Elisha Scott Loomis (1852-1940) (Figure 7), an eccentric mathematics teacher from Ohio, spent a lifetime collecting all known proofs of the Pythagorean Theorem and writing them up in 


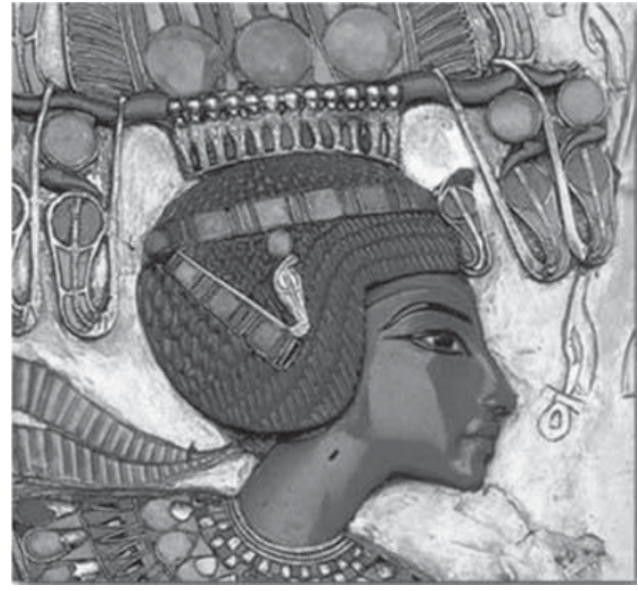

Figure 6 'King Tut' (Not the famous burial mask).

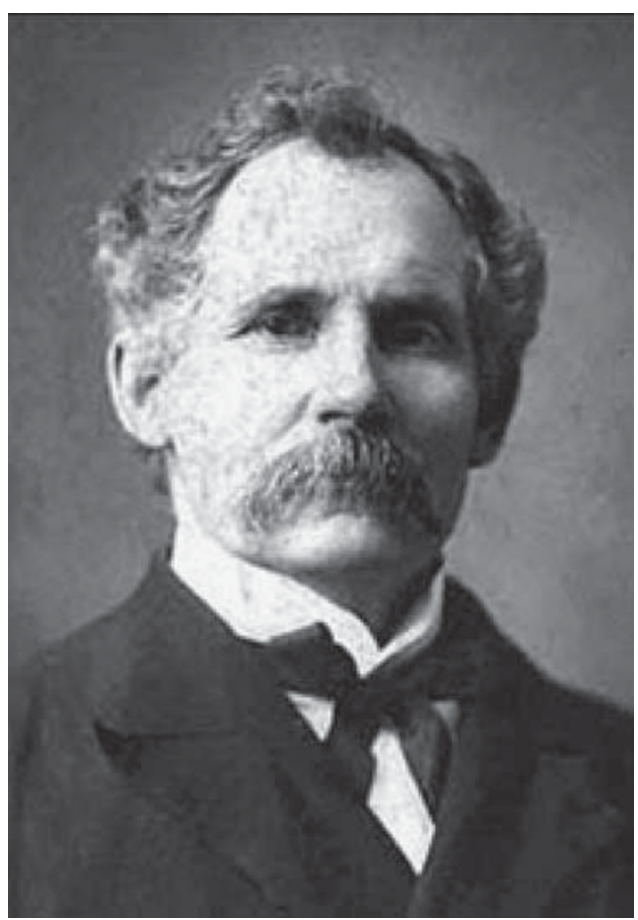

Figure 7: Elisha Scott Loomis.

The Pythagorean Proposition, a compendium of 371 proofs. The manuscript was prepared in 1907 and published in 1927. A revised second edition appeared in 1940, and this was reprinted by the National Council of Teachers of Mathematics in 1968 as part of its 'Classics in Mathematics Education' series. Loomis received literally

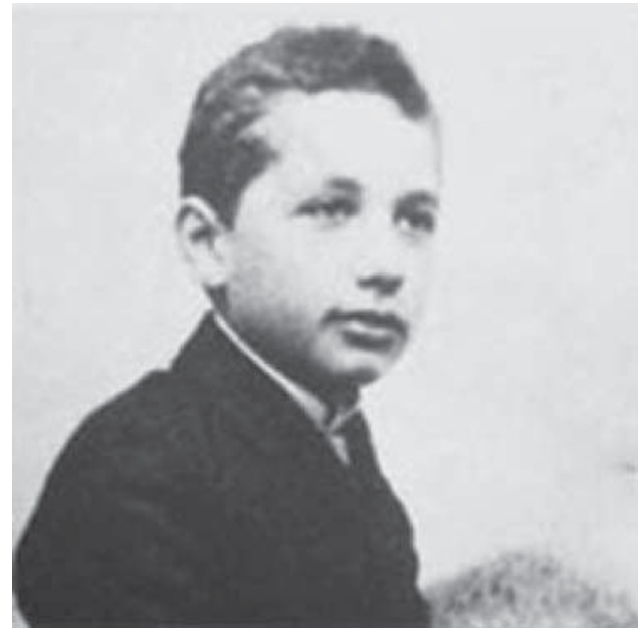

Figure 8: Albert Einstein, age 12.

hundreds of new proofs from after his book was released up until his death, but he could not keep up with his compendium. As for the exact number of proofs, no one is sure how many there are. Surprisingly, geometricians often find it quite difficult to determine whether certain proofs are in fact distinct proofs. Befitting of someone who collects solutions of the Pythagorean Theorem (I belittle neither the effort nor its value), Loomis, known for living an orderly life, extended his writing to his own obituary in 1934, which he left in a letter headed 'For the Berea Enterprise immediately following my death'. He died on 11 December 1940, and the obituary was published as he had written it, except for the date of his death and the addresses of some of his survivors. ${ }^{21}$

\section{A 12-YEAR-OLD EINSTEIN 'PROVES' THE PYTHAGOREAN THEOREM}

According to his autobiography, a preteen Albert Einstein (Figure 8)

devised a new 'proof' (he was careful to put the word in quotation marks, evidently not wishing to take credit for it) of the Pythagorean Theorem based on the properties of similar triangles. Many known proofs use similarity arguments, but this one is notable for its elegance, simplicity and the sense that it reveals the connection between length and area that is at the heart of the theorem. ${ }^{22}$ 
About his 'holy geometry book', Einstein in his autobiography says:

At the age of 12, I experienced a second wonder of a totally different nature: in a little book dealing with Euclidean plane geometry, which came into my hands at the beginning of a school year. Here were assertions, as for example the intersection of the three altitudes of a triangle in one point, which - though by no means evident - could nevertheless be proved with such certainty that any doubt appeared to be out of the question. This lucidity and certainty made an indescribable impression upon me. For example I remember that an uncle told me the Pythagorean Theorem before the holy geometry booklet had come into my hands. After much effort I succeeded in 'proving' this theorem on the basis of the similarity of triangles ... for anyone who experiences [these feelings] for the first time, it is marvelous enough that man is capable at all to reach such a degree of certainty and purity in pure thinking as the Greeks showed us for the first time to be possible in geometry. ${ }^{23}$

\section{EINSTEIN'S CHILDHOOD FASCINATION WITH THE PYTHAGOREAN THEOREM BEARS FRUIT}

Einstein (Figure 9) used the Pythagorean

Theorem in the Special Theory of Relativity (in a four-dimensional form), and in a vastly expanded form in the General Theory of Relatively. The following excerpts are worthy of inclusion.

\section{Pythagorean Theorem in the Special Theory of Relativity (1905)}

Albert Einstein's Metric equation is simply Pythagoras' Theorem applied to the three spatial co-ordinates and equating them to the displacement of a ray of light.

Special relativity is still based directly on an empirical law, that of the constancy of the velocity of light.

$\mathrm{d} x^{2}+\mathrm{d} y^{2}+\mathrm{d} z^{2}=(c \mathrm{~d} t)^{2}$ where $c \mathrm{~d} t$ is the distance traveled by light $c$ in time $\mathrm{d} t$. The fact that such a metric is called Euclidean is connected with the following. The postulation of such a metric in a three-dimensional continuum is fully

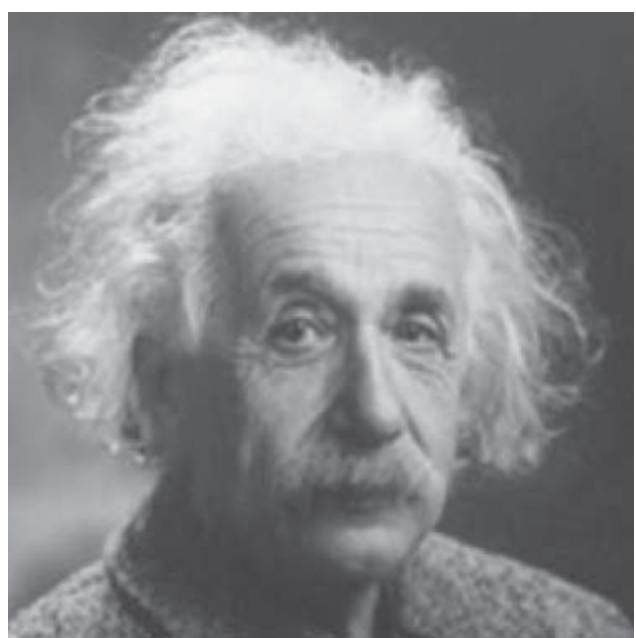

Figure 9: Einstein.

equivalent to the postulation of the axioms of Euclidean Geometry. The defining equation of the metric is then nothing but the Pythagorean Theorem applied to the differentials of the co-ordinates.

In the special theory of relativity those co-ordinate changes (by transformation) are permitted for which also in the new co-ordinate system the quantity $(c \mathrm{~d} t)^{2}$ (fundamental invariant $\mathrm{d} S^{2}$ ) equals the sum of the squares of the co-ordinate differentials. Such transformations are called Lorentz transformations. ${ }^{24}$

\section{Pythagorean Theorem in the General Theory of Relativity (1915)}

From the latest results of the theory of relativity, it is probable that our three-dimensional space is also approximately spherical, that is, that the laws of disposition of rigid bodies in it are not given by Euclidean geometry, but approximately by spherical geometry. According to the general theory of relativity, the geometrical properties of space are not independent, but they are determined by matter. I wished to show that space time is not necessarily something to which one can ascribe to a separate existence, independently of the actual objects of physical reality. Physical objects are not in space, but these objects are spatially extended. In this way the concept 'empty space' loses its meaning. The fact that such a metric is called 
Euclidean is connected with the following. The postulation of such a metric in a threedimensional continuum is fully equivalent to the postulation of the axioms of Euclidean Geometry. The defining equation of the metric is then nothing but the Pythagorean Theorem applied to the differentials of the co-ordinates. ${ }^{25}$

\section{The Theory of Relativity and the Pythagorean Theorem}

The above excerpts - from the genius himself - precede any other person's narrative of the Theory of Relativity and the Pythagorean Theorem. Accordingly, I now provide a less demanding excerpt, albeit one that addresses the effects of the Special and General theories of relativity.

... the most important effects of special and general theory of relativity can be understood in a simple and straightforward way. The system of units in which the speed of light $\mathrm{c}$ is the unit of velocity allows to cast all formulas in a very simple form. The Pythagorean Theorem graphically relates energy, momentum and mass. ${ }^{26}$

\section{EUCLID'S ELEMENTS}

Euclid of Alexandria was a Greek mathematician (Figure 10), and is often referred to as the Father of Geometry. The date and place of Euclid's birth, and the date and circumstances of his death, are unknown, but it is thought that he lived circa 300 BCE. His work Elements, which includes books and propositions, is the most successful textbook in the history of mathematics. In it, the principles of what is now called Euclidean Geometry were deduced from a small set of axioms.

When Euclid wrote his Elements around 300 BCE, he gave two proofs of the Pythagorean Theorem: The first, Proposition 47 of Book I, relies entirely on the area relations and is quite sophisticated; the second, Proposition 31 of Book $\mathrm{VI}$, is based on the concept of proportion and is much simpler. The first could not be Pythagoras' own proof because geometry was simply not advanced enough at that time. He may have used Book VI Proposition 31, but, if so, his proof

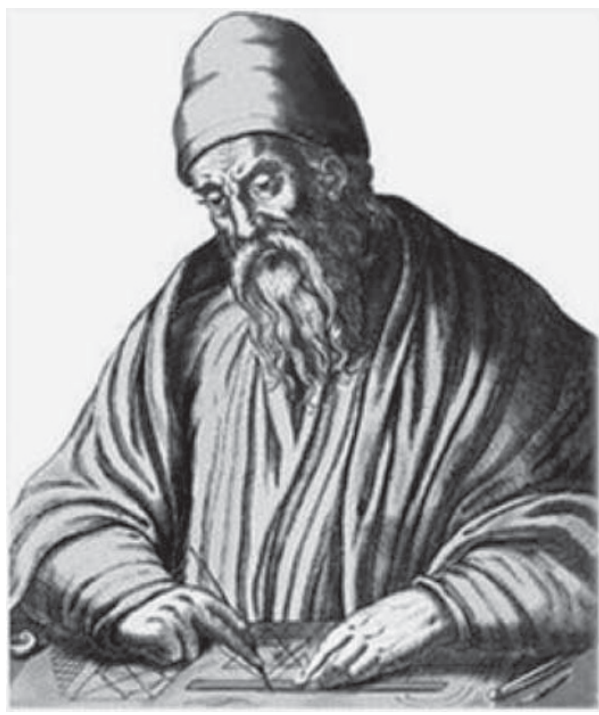

Figure 10: Artist's impression of Euclid.

was deficient, because the complete theory of Proportions was only developed by Eudoxus, who lived almost two centuries after Pythagoras. ${ }^{27}$

Euclid's Elements furnishes the first and, later, the standard reference in geometry. It is a mathematical and geometric treatise consisting of 13 books. It comprises a collection of definitions, postulates (axioms), propositions (theorems and constructions) and mathematical proofs of the propositions.

Elements' table of contents is shown in Figure $11 .^{28}$ One of the oldest surviving fragments of Euclid's Elements is shown in Figure 12. ${ }^{29}$

Euclid provided two very different proofs, stated below, of the Pythagorean Theorem. Euclid was the first to mention and prove Book I, Proposition 47, also known as I 47 or Euclid I 47. This is probably the most famous of all the proofs of the Pythagorean proposition.

Book VI, Proposition 31: In right-angled triangles the figure on the side opposite the right angle equals the sum of the similar and similarly described figures on the sides containing the right angle.

Book I, Proposition 47: In right-angled triangles the square on the side opposite the right angle equals the sum of the squares on the sides containing the right angle. 


\section{Euclid's Elements}

\section{Table of Contents}

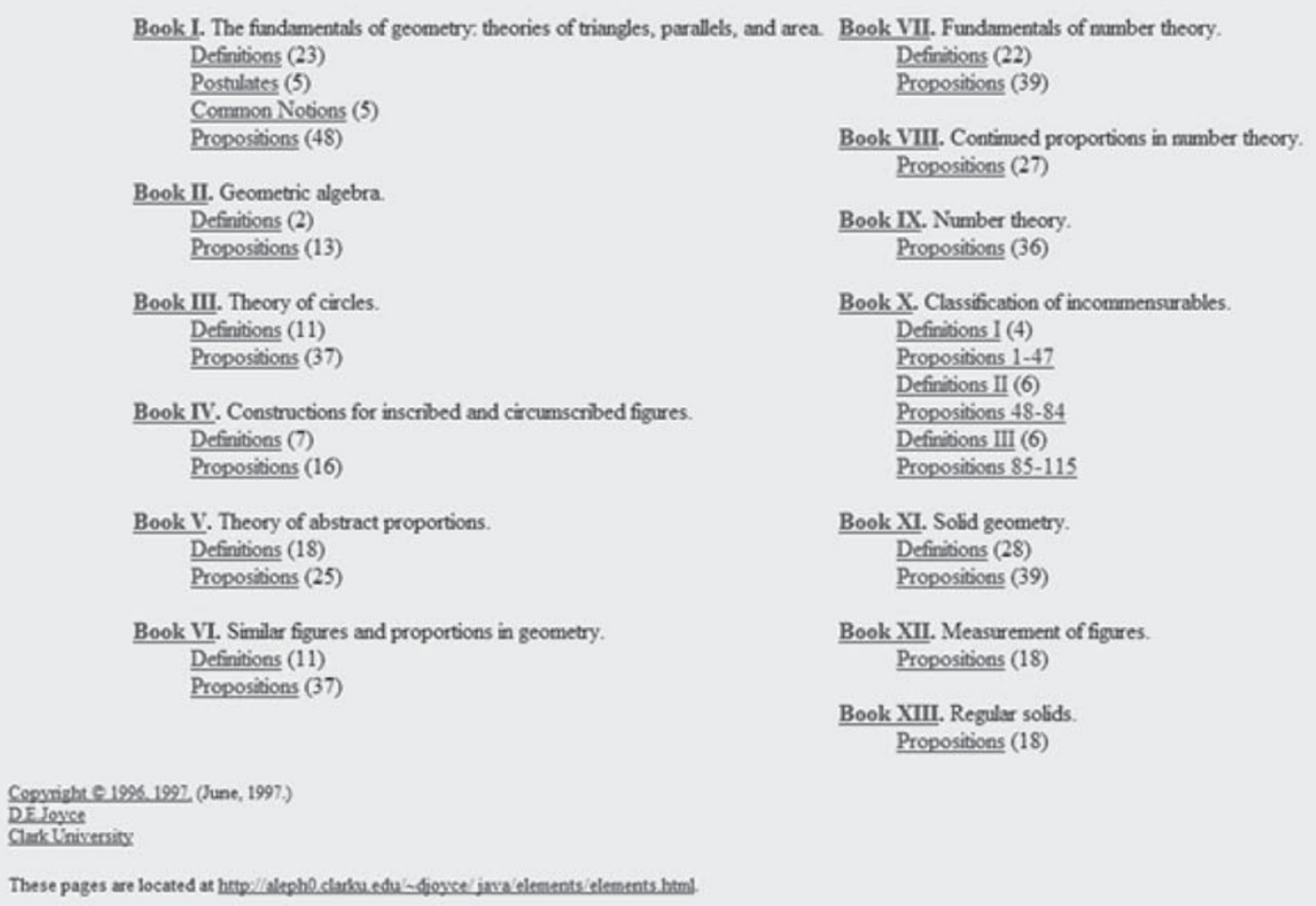

Figure 11: Euclid's elements.

Euclid I 47 is often called the Pythagorean Theorem, called so by Proclus - a Greek philosopher who became head of Plato's Academy and is important mathematically for his commentaries on the work of other mathematicians - and others centuries after Pythagoras and even centuries after Euclid. ${ }^{30}$

Although many of the results in Elements originated with earlier mathematicians, one of Euclid's accomplishments was to present them in a single, logically coherent framework, making them easy to use and easy to reference, including a system of rigorous mathematical proofs that remains the basis of mathematics twenty-three centuries later.

Although best known for its geometric results, Elements also includes number theory. It considers the connection between perfect numbers and Mersenne primes, the infinitude of prime numbers and the Euclidean algorithm for finding the greatest common divisor of two numbers. ${ }^{31}$

The geometrical system described in the Elements was long known simply as geometry, and was considered to be the only geometry possible. Today, however, this system is often referred to as Euclidean Geometry to distinguish it from other so-called Non-Euclidean geometries that mathematicians discovered in the nineteenth century. ${ }^{32}$

\section{A simple proof of the Pythagorean Theorem}

At this point in my plotting of the 4000-year-old story of Pythagoras, I feel it is fitting to present one proof of the famous theorem. For me, the 


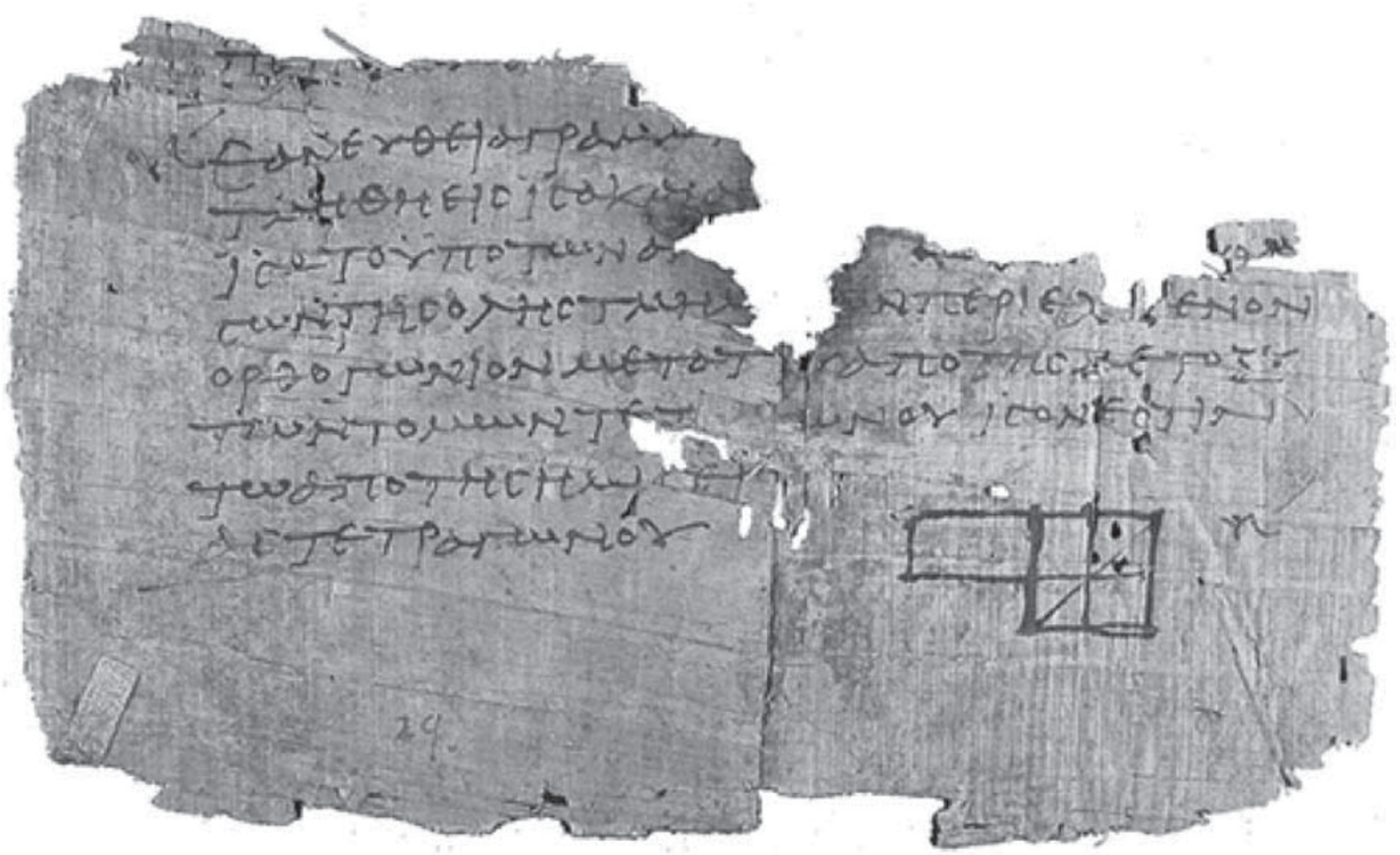

Figure 12: One of the oldest surviving fragments of Euclid's elements.

simplest proof among the dozens of proofs that I read in preparing this article is that shown in Figure $13 .{ }^{33-35}$

1. Start with four copies of the same triangle. Three of these have been rotated $90^{\circ}, 180^{\circ}$ and $270^{\circ}$, respectively. Each has area $a b / 2$. (See upper part of Figure 13.)

2. Combine the four triangles to form an upright square with the side $(a+b)$, and a tilted square-hole with the side $c$. (See lower part of Figure 13.)

3. Compute the area of the big square in two ways:

- The direct area of the upright square is $(a+b)^{2}$

- The collective-four-copies area of the titled square-hole is $4(a b / 2)+c^{2}$

4. $(a+b)^{2}=4(a b / 2)+c^{2}$

QED (abbreviation, Latin, Quod Erat Demonstrandum: that which was to be demonstrated.)

\section{A GENERALIZED VERSION OF THE PYTHAGOREAN THEOREM}

In the seventeenth century, Pierre de Fermat (1601-1665) (Figure 14) investigated the following problem: for which values of $n$ are there integer solutions to the equation

$$
x^{n}+y^{n}=z^{n}
$$

It is known that when $n=2$ then an integer solution exists from the Pythagorean Theorem. Fermat conjectured that there were no non-zero integer solutions for $x$ and $y$ and $z$ when $n$ was greater than 2. He did not leave a proof, though. Instead, in the margin of a textbook, he wrote that he knew that this relationship was not possible, but he did not have enough room on the page to write it down. His conjecture became known as Fermat's Last Theorem. This may appear to be a simple problem on the surface, but it was not until 1993 when Andrew Wiles of Princeton University finally proved the 350-year-old marginalized theorem, which appeared on the front page of the New York Times. ${ }^{36}$

Today, Fermat is thought of as a number theorist, in fact perhaps the most famous number theorist who ever lived. It is therefore surprising to find that Fermat was a lawyer, and only an amateur mathematician. Also surprising 


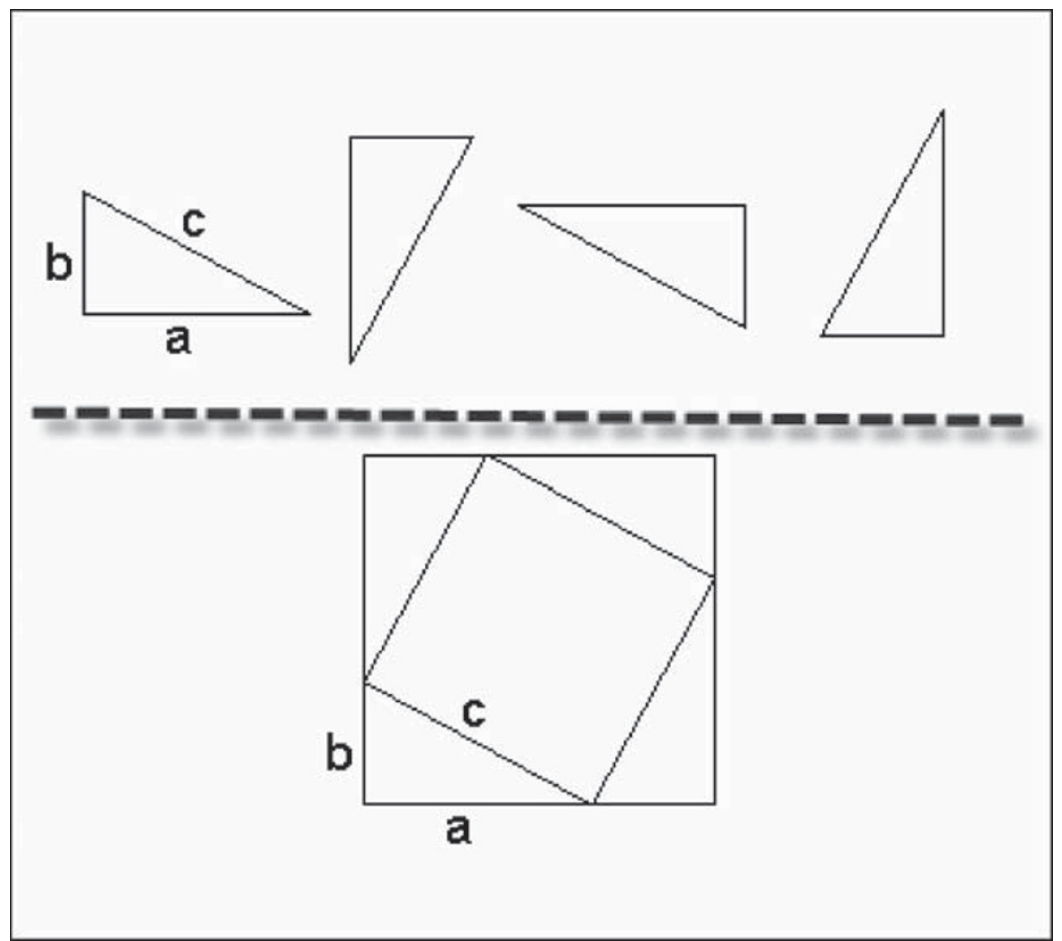

Figure 13: A simple proof of the Pythagorean Theorem.

is the fact that he published only one mathematical paper in his life, and that was an anonymous paper written as an appendix to a colleague's book.

Because Fermat refused to publish his work, his friends feared that it would soon be forgotten unless something was done about it. His son Samuel undertook the task of collecting Fermat's letters and other mathematical papers, comments written in books and so on with the goal of publishing his father's mathematical ideas. Samuel found the marginal note (the proof could not fit on the page) in his father's copy of Diophantus's Arithmetica. In this way the famous Last Theorem came to be published. ${ }^{37}$

\section{FERMAT'S LAST THEOREM: SOLVED}

Sir Andrew John Wiles, KBE (Knight Commander of the Order of the British Empire), mathematician and professor at Princeton University, specializing in number theory, is forever famous for proving Fermat's Last Theorem (Figure 15).

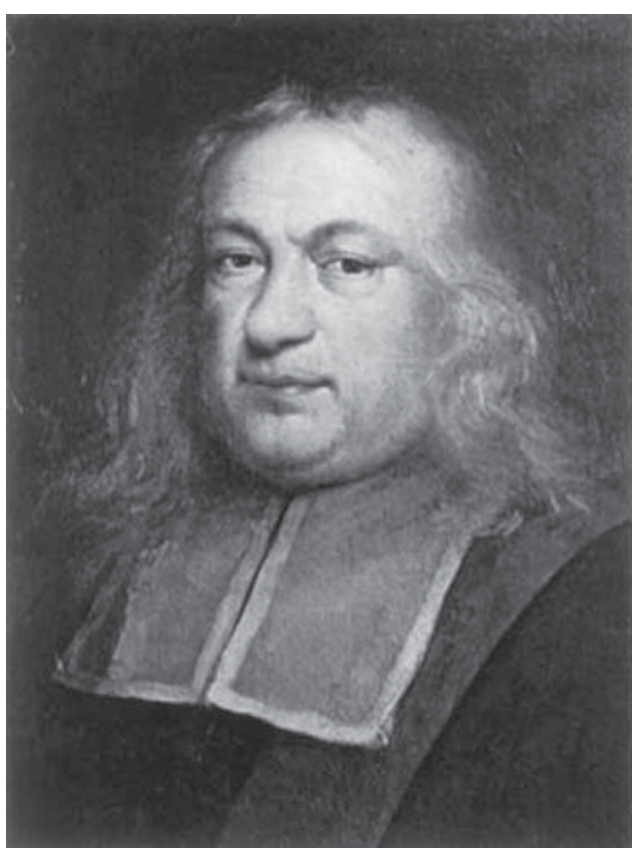

Figure 14: Pierre de Fermat.

Andrew Wiles was born in Cambridge, England in 1953, and attended King's College School, Cambridge (where his mathematics 


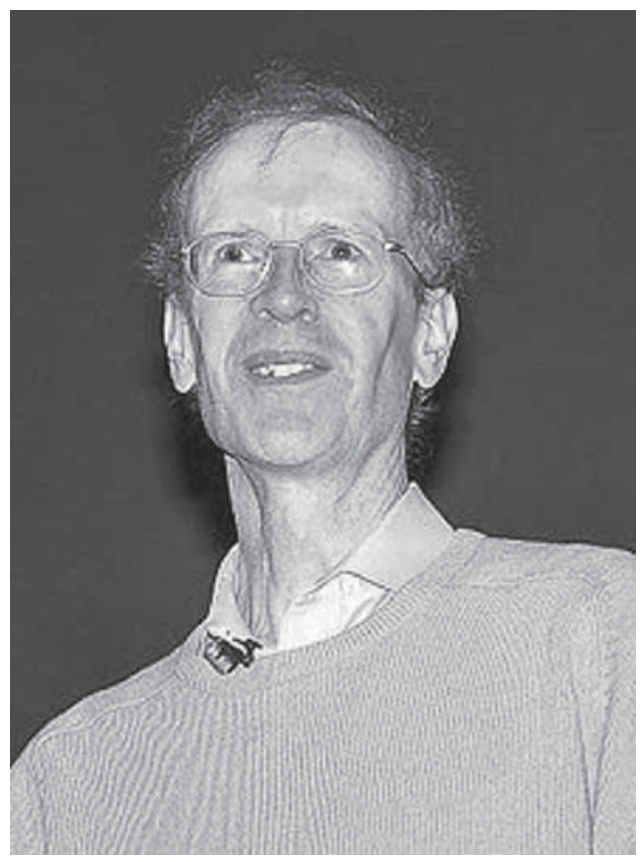

Figure 15: Sir Andrew Wiles.

teacher David Higginbottom first introduced him to Fermat's Last Theorem). He earned his BA in 1974 after study at Merton College, Oxford, and a $\mathrm{PhD}$ in 1980 after research at Clare College, Cambridge. His graduate research was guided by John Coates beginning in the summer of 1975 . Together they worked on the arithmetic of elliptic curves with complex multiplication using the methods of Iwasawa theory.

He further worked with Barry Mazur on the main conjecture of Iwasawa theory over $Q$ and soon afterwards generalized this result to totally real fields. Taking approximately 7 years to complete the work, Wiles was the first person to prove Fermat's Last Theorem, earning him a place in history. Andrew Wiles' most famous mathematical result is that all rational semi-stable elliptic curves are modular, which, in particular, implies Fermat's Last Theorem.

Wiles was introduced to Fermat's Last Theorem at the age of 10 . He tried to prove the theorem using textbook methods and later studied the work of mathematicians who had tried to prove it. When he began his graduate studies, he stopped trying to prove the theorem and began studying elliptic curves under the supervision of John Coates.

In the 1950s and 1960s, a connection between elliptic curves and modular forms was conjectured by the Japanese mathematician Goro Shimura based on some ideas that Yutaka Taniyama posed. In the West, this conjecture became well known through a paper by André Weil. With Weil giving conceptual evidence for it, it is sometimes called the Shimura-Taniyama-Weil conjecture. It states that every rational elliptic curve is modular. The full conjecture was proven by Christophe Breuil, Brian Conrad, Fred Diamond and Richard Taylor in 1998 using many of the methods that Andrew Wiles used in his 1995 published papers. ${ }^{38}$

\section{CONCLUSION}

I provide the story of Pythagoras and his famous theorem by discussing the major plot points of a 4000-year-old fascinating story in the history of mathematics, worthy of recounting even for the math-phobic reader. It is more than a math story, as it tells a history of two great civilizations of antiquity rising to prominence 4000 years ago, along with historic and legendary characters, who not only define the period, but whose life stories individually are quite engaging.

The intriguing plot points of the story are:

1. Pythagoras is immortally linked to the discovery and proof of a theorem, which bears his name - even though there is no evidence of his discovering and/or proving the theorem.

2. Greek mathematician Euclid, referred to as the Father of Geometry, lived during the period of time about $300 \mathrm{BCE}$, when he was most active. His work Elements is the most successful textbook in the history of mathematics. Euclid I 47 is often called the Pythagorean Theorem, called so by Proclus, a Greek philosopher who became head of Plato's Academy and is important mathematically for his commentaries on the work of other mathematicians centuries after Pythagoras and even centuries after Euclid. 
3. There is concrete (not Portland cement, but a clay tablet) evidence that indisputably indicates that the Pythagorean Theorem was discovered and proven by Babylonian mathematicians 1000 years before Pythagoras was born.

4. So many people, young and old, famous and not famous, have touched the Pythagorean Theorem. The eccentric mathematics teacher Elisha Scott Loomis spent a lifetime collecting all known proofs and writing them up in The Pythagorean Proposition, a compendium of 371 proofs. The manuscript was published in 1927, and a revised, second edition appeared in 1940. As for the exact number of proofs, no one is sure how many there are. Surprisingly, geometricians often find it quite difficult to determine whether some proofs are in fact distinct proofs. Moreover, the theorem seemingly has no ending, as every year students, academicians and problem solvers with a mathematical bent tackle the theorem in an attempt to add new and innovative proofs.

5. In addition, many people's lives have been touched by the Pythagorean Theorem. A 12-year-old Albert Einstein was touched by the earthbound spirit of the Pythagorean Theorem. The wunderkind provided a proof that was notable for its elegance and simplicity. However, the spirit of the Pythagoras' Theorem was not finished with young Einstein: two decades later he used the Pythagorean Theorem in the Special Theory of Relativity (in a fourdimensional form), and in a vastly expanded form in the General Theory of Relativity. That Einstein used Pythagorean Theorem for his Relativity would be enough to show Pythagorean Theorem's value, or importance to the world. But, people continued to find value in the Pythagorean Theorem, namely, Wiles.

6. The theorem's spirit also visited another youngster, a 10-year-old British Andrew Wiles, and returned two decades later to an unknown Professor Wiles. Young Wiles tried to prove the theorem using textbook methods, and later studied the work of mathematicians who had tried to prove it.
When he began his graduate studies, he stopped trying to prove the theorem and began studying elliptic curves, which provided the path for proving Fermat's Theorem, the news of which made to the front page of the New York Times in 1993. Sir Andrew Wiles will forever be famous for his generalized version of the Pythagoras Theorem.

\section{REFERENCES}

1 Journal Physics World (2004), as reported in the New York Times, Ideas and Trends, 24 October 2004, p. 12.

2 Maor, E. (2007) The Pythagorean Theorem, A 4,000-Year History. Princeton, NJ: Princeton University Press, p. xii.

3 Leonardo da Vinci (15 April 1452 - 2 May 1519) was an Italian polymath (someone who is very knowledgeable), being a scientist, mathematician, engineer, inventor, anatomist, painter, sculptor, architect, botanist, musician and writer. Leonardo has often been described as the archetype of the Renaissance man, a man whose unquenchable curiosity was equaled only by his powers of invention. He is widely considered to be one of the greatest painters of all time and perhaps the most diversely talented person ever to have lived. 'The scope and depth of his interests were without precedent ... . His mind and personality seems to us superhuman, the man himself mysterious and remote', http://en.wikipedia.org/wiki/ Leonardo_da_Vinci.

4 Loomis, E. S. (1927) The Pythagorean Proportion, A revised, second edition appeared in 1940, reprinted by the National Council of Teachers of Mathematics in 1968 as part of its 'Classics in Mathematics Education' series.

5 http://math.colgate.edu/faculty/dlantz/Pythpfs/Garfldpf.html.

6 Maor, E. (2007) The Pythagorean Theorem, A 4,000-Year History. Princeton, NJ: Princeton University Press, p. 17.

7 http://www-groups.dcs.st-and.ac.uk/ history/Biographies/ Pythagoras.html.

8 http://www.themiddlechamber.com/Pythagoras_Freemqwonary.pdf.

9 http://www.davesabine.com/Music/Articles/Pythagoras MathematicalTheoruminMusic/tabid/169/Default.aspx.

10 A rational number is a number that can be expressed as a fraction or ratio (rational). The numerator and the denominator of the fraction are both integers. When the fraction is divided out, it becomes a terminating or repeating decimal. (The repeating decimal portion may be one number or a billion numbers.) Rational numbers can be ordered on a number line.

11 An irrational number cannot be expressed as a fraction. Irrational numbers cannot be represented as terminating or repeating decimals. Irrational numbers are non-terminating, non-repeating decimals. Examples of irrational numbers are: square root of $2=1.414213562 \ldots ; \pi=3.141592654 \ldots$.

12 http://tubulcain420.blogspot.com/2008/11/life-and-philosophyof-pythagoras.html.

13 http://www.bible-history.com/babylonia/BabyloniaGeography.htm.

14 Maor, E. (2007) The Pythagorean Theorem, A 4,000-Year History. Princeton, NJ: Princeton University Press, p. 5.

15 http://www.bible-history.com/babylonia/BabyloniaGeography .htm. 
16 Copyright to the images of YBC 7289 belongs to photographer Bill Casselman, http://www.math.ubc.ca/ cass/Euclid/ybc/ybc.html.

$17 \mathrm{http} / / /$ en.wikipedia.org/wiki/Square_root_of_2\#Geometric_proof.

18 http://www-groups.dcs.st-and.ac.uk/ history/Biographies/ Pythagoras.html.

19 http://www.thebigview.com/greeks/pythagoras.html.

$20 \mathrm{http} / / /$ en.wikipedia.org/wiki/Tutankhamun.

21 http://www.bgsu.edu/departments/math/Ohio-section/bicen/ esloomis.html.

22 http://demonstrations.wolfram.com/EinsteinsMostExcellentProof/.

23 Schilpp, P. A. (ed.) (1951) Albert Einstein: Philosopher-Scientist, pp. $9-11$.

24 The excerpted section on Pythagoras' Theorem and its use in Einstein's Relativity is from the article Physics: Albert Einstein's Theory of Relativity.

25 http://www.spaceandmotion.com/Physics-Albert-EinsteinTheory-Relativity.htm.

26 Okun, L. B. (2008) The theory of relativity and the Pythagorean theorem. Physics-Uspekhi 51: 622.

27 http://en.wikipedia.org/wiki/Euclid.
28 http://en.wikipedia.org/wiki/Euclid\%27s_Elements\#Contents_ of_the_books.

29 http://en.wikipedia.org/wiki/File:Oxyrhynchus_papyrus_with_ Euclid\%27s_Elements.jpg.

30 http://www-groups.dcs.st-and.ac.uk/ history/Mathematicians/ Proclus.html.

31 Mersenne number is a positive integer that is one less than a power of two: $M_{n}=2^{n}-1$.

32 http://www-groups.dcs.st-and.ac.uk/ history/HistTopics/NonEuclidean_geometry.html.

33 http://www.cut-the-knot.org/pythagoras/index.shtml.

34 http://www.sunsite.ubc.ca/DigitalMathArchive/Euclid/java/ html/babylon.html (Oldest known proof of Pythagorean Theorem).

35 http://mathworld.wolfram.com/PythagoreanTheorem.html.

36 http://www-groups.dcs.st-and.ac.uk/ history/Biographies/ Fermat.html.

37 http://www.joh.cam.ac.uk/library/special_collections/early_ books/pix/fermat.htm.

38 http://en.wikipedia.org/wiki/Andrew_Wiles. 